\section{Three RFLPs at the D8S49 locus}

Bernhard Horsthemke*, Ruth Burdiek and HermannJosef Lüdecke

Institut für Humangenetik, Universitätsklinikum Essen, Hufelandstrasse 55, D-4300 Essen 1, FRG

Source/Description: L40 is a 134 bp single copy genomic DNA fragment cloned into pUC13. The clone was isolated by microdissection and microcloning of the Langer-Giedion syndrome chromosome region (LGCR).

Polymorphisms: LA0 identifies three two-allele polymorphisms:

$\begin{array}{llll}\text { SacI } & 23.0 \mathrm{~kb} \mathrm{A1} & \text { AspI } & 8.0 \mathrm{~kb} \mathrm{B1} \\ & 30.0 \mathrm{~kb} \mathrm{~A} 2 & & 13.5 \mathrm{~kb} \mathrm{~B} 2 \\ \text { BclI } & 1.9 \mathrm{~kb} \mathrm{C1} & & \end{array}$

$15.0 \mathrm{~kb} \mathrm{C} 2$

Two constant bands of $3.5 \mathrm{~kb}$ and $1.6 \mathrm{~kb}$ are seen in the AspI digest.

$\begin{array}{lll}\text { Frequencies: } & & \\ \text { Allele } & \text { Frequency } & \text { Number of Chromosomes } \\ \text { A1 } & 0.69 & 32 \\ \text { A2 } & 0.31 & \\ \text { B1 } & 0.67 & 30 \\ \text { B2 } & 0.33 & \\ \text { C1 } & 0.63 & 24 \\ \text { C2 } & 0.37 & \end{array}$

Not Polymorphic For: AccI, Asp700, BanI, BanII, BamHI, BglI, BglII, BstNI, BstXI, DraI, EcoRI, EcoRV, HindII, HindIII, Hinfl, HgiAI, HpaI, KpnI, MspI, NciI, PstI, PvuII, RsaI, Sau96I, StuI, TaqI, XbaI in a screen of six unrelated Caucasian individuals.

Chromosomal Localization: Mapped to 8q23.2-q24.11 by quantitative Southern blot hybridization of DNA from patients with Langer-Giedion syndrome that have cytogenetically visible deletions.

Mendelian Inheritance: Autosomal codominant segregation was observed in two large Caucasian families.

Probe Availability: Available for collaboration: contact B.Horsthemke.

Other Comments: Insert can be released from the vector by EcoRI digestion.

Acknowledgements: Supported by the Deutsche Forschungsgemeinschaft.

Reference: Lüdecke et al. (1989) Nature 338, 348-350.

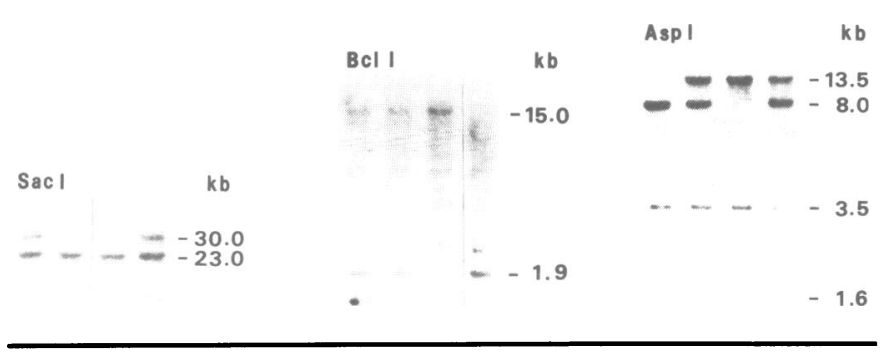

* To whom correspondence should be addressed

\section{Two RFLPs at the D8S50 locus}

Bernhard Horsthemke*, Ruth Burdiek and HermannJosef Lüdecke

Institut für Humangenetik, Universitätsklinikum Essen, Hufelandstrasse 55, D-4300 Essen 1, FRG

Source/Description: L47 is a 200 bp single copy genomic DNA fragment cloned into pUC13. The clone was isolated by microdissection and microcloning of the Langer-Giedion syndrome chromosome region (LGCR).

Polymorphisms: L47 identifies two two-allele polymorphisms:

BanII $\quad 9.6 \mathrm{~kb} \mathrm{A1} \quad$ MspI $18.0 \mathrm{~kb} \mathrm{B1}$

$$
7.4 \mathrm{~kb} \mathrm{A2} \quad 13.5 \mathrm{~kb} \mathrm{~B} 2
$$

Frequencies: Estimated from 30 chromosomes

Allele Frequency

A1 0.9

A2 0.1

B1 0.9

B2 0.1

Not Polymorphic For: AccI, Avall, AspI, Asp700, BanI, BamHI, BclI, BglI, BglII, BstNI, BstXI, DraI, EcoRI, EcoRV, HindII, HindIII, HinfI, HgiA1, HpaI, KpnI, NciI, PstI, PvuII, RsaI, SacI, Sau96I, StuI, TaqI, XbaI in a screen of six unrelated Caucasian individuals.

Chromosomal Localization: Mapped to $8 \mathrm{q} 23.2-\mathrm{q} 24.11$ by quantitative Southern blot hybridization of DNA from patients with Langer-Giedion syndrome that have cytogenetically visible deletions.

Mendelian Inheritance: Autosomal codominant segregation was observed in two large Caucasian families.

Probe Availability: Available for collaboration: contact B.Horsthemke.

Other Comments: Insert can be released from the vector by EcoRI digestion.

Acknowledgements: Supported by the Deutsche Forschungsgemeinschaft.

Reference: Lüdecke et al. (1989) Nature 338, 348-350.

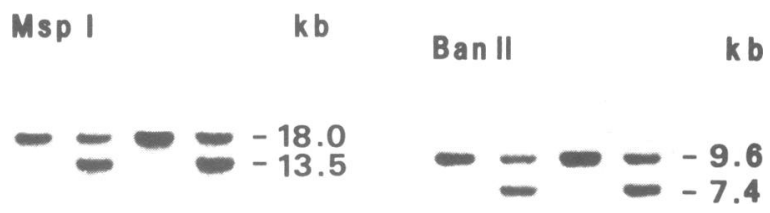

* To whom correspondence should be addressed 